\title{
Data Animator: Visualization of two- and three dimensional data as computer generated animations
}

\author{
Efraim Halfon \\ National Water Research Institute \\ Canada Centre for Inland Waters, Burlington, Ontario, Canada L7R 4A6,Tel. 905- \\ 336-4917; Fax.905-336-4972, E-mail: EFRAIM.HALFON@ CCIW.CA
}

\begin{abstract}
Data Animator V1.0 is a powerful, easy-to-use scientific visualization package. Its main purpose is to generate animations from any data set collected over time. Geographical references such as a shore, bathymetry information, etc., may be added for additional clarity. Visualization of data as animations can greatly simplify the interpretation of field measurements. Data Animator is designed (but not restricted) to display data collected in aquatic environments, lakes, rivers, estuaries, oceans, etc., in a clear, concise way using colours to represent ranges of data values. Data sets can also be displayed as static images (keyframes). A graphic user interface (GUI) allows the user to choose a viewpoint, fonts, colour palette, data and keyframes. Point-and-click mouse operations allow the user to manipulate many features, with immediate on-screen feedback. This GUI allows the user to quickly design an attractive animation (or single frame) to exact specifications. Animations are generated by defining 'keyframes' of known data, each located at a specific point in time. The program can then interpolate over time, between keyframes, to create smoothly animated transitions (in-between frames). Two types of graphs can be rendered with Data Animator. 'Plane' type graphs are horizontal slices at a depth specified by the user. 'Transect' type graphs are vertical slices along a straight line defined by the user. Data Animator can make use of both shore outline information and three-dimensional bathymetry information. This allows for the generation of realistic-looking graphs which follow the shape of the aquatic environment. A typical animation, in FLC format, can be displayed at 30 frames per second. Animations can be displayed on a computer monitor or transferred to video tape.
\end{abstract}

Keywords

Scientific visualization, computer graphics, animation, data analysis, lake, aquatic system, 3D, 2D, limnology, oceanography 
INTRODUCTION

Data collected in three dimensions are commonly available from limnological research. A ship collects data at several stations, and at many depths. When data are collected over time at many locations, a four dimensional picture surfaces. The fourth dimension is temporal. A computer generated animation can visualize the dynamics over time of three dimensional data.

While in the medical field (Herman and Liu, 1979; Sunguroff and Greenberg, 1978; Kaufmann, 1991) computer imaging is important for diagnostics and planning operations, in limnology computer imaging might be important to visualize the movement of water masses, the development of algal blooms, episodes of oxygen depletion, etc. Advanced methods of computer graphics can help scientists and water managers alike (Halfon, 1989).

The purpose of Data_Animator is to display data as computer generated animations. The lake bathymetry and topography are also an integral part of the package. Thus, the main function of the program is to generate (render) computer animations from data. This main advantage of this program is that it is user friendly and it can be learned in about an hour. Other more comprehensive visualization programs can only run on expensive workstations with a long learning time, while Data Animator runs on PCs and its use can be learned in about an hour. Best performance can be obtained on Pentium based machines. Its animations can be viewed on the computer or transferred on video.

Scientific visualization (Halfon, 1995) is a new and rapidly expanding field of research. Visualization permits a dynamic comparison of data collected over a season or over several years. Understanding of the data is facilitated by this tool. Computer animation, computer graphics, and video technology can also be used to present both to the general public and to specialized scientific audiences research result on aquatic environments. The use of animation can be expanded to include preparation of interactive information tools, such as CD-ROMs. Data Animator (Halfon et al., 1993, 1994; Halfon and Howell, 1995; Halfon and Tartari, 1995) has been used to interpret temperature and oxygen data collected in Hamilton Harbour, Lake Ontario and Lake Orta, an alpine Lake in Italy.

Limnological data are quite sparse in space. While a catscan (a three dimensional x-ray) might assemble information with a size of $512 \times 512 \times 512$ pixels within hours, a limnologist would be hard pressed in collecting data at tens of stations, and at many depths, in less than a few days of field work, plus laboratory time. Lack of data creates problems of interpolation and extrapolation when computer animations are created. Also, to display three dimensional data collected in a lake, the lake bathymetry must be included to show the relation of the data to the bottom, and to the shoreline. In computer graphics, this relation takes the form of a mask. No data are shown on land. The development of this three dimensional mask is computationally challenging.

\subsection{The grouping facility}

One very important option is what we call 'grouping'. Grouping allows the user to separate regions which are physically close but logically separate, such as two bodies of water connected only by a narrow channel. When grouping is needed the number of groups can be defined as two, or greater. The grouping facility allows the user to define regions of a graph to be interpolated independently from each other. This is done by simply drawing polygons on a map of the region. Points which fall inside a polygon are interpolated using only the data that are also found within the polygon. The grouping facility has been developed to independently render different areas. The rationale is that some areas are not collected in reality and therefore the interpolation process should only use some stations and not 
others. The word grouping has been chosen since it means to divide the area to be rendered in different groups. The first group by default is zero (this number is for internal use of the program only).

\section{DATA REQUIREMENTS}

A standard input format was designed to incorporate the data and its geographical and temporal reference. The format is ASCII. Data collected in Hamilton Harbour, Lake Ontario, are used here as an example. The first column is concentration of dissolved oxygen, the second column represents temperature data, the third column is depth from the surface, the fourth is conductivity, followed by transmissivity and $\mathrm{pH}$. The order of the data in the column is immaterial. Depth is just another variable, thus is it possible to visualize, for example, oxygen vs. temperature, $\mathrm{pH}$ vs conductivity, etc. Data files must be organized by sample time. All samples taken within the same period are included in one file. The date and time are read from the first sample in the file. Each file can contain data from several sample sites.

\section{Site Header \\ Site Data \\ "\#EOF" String}

The following is an example of a sample data file (90051):

lat long date station number

\begin{tabular}{|c|c|c|c|}
\hline \multicolumn{4}{|c|}{431828794645900528908} \\
\hline 13.4702 & 12.8028 & .1666 & 55.3785 \\
\hline 13.4497 & 12.8020 & .2265 & 55.6547 \\
\hline 13.8910 & 12.7128 & .4615 & 59.3252 \\
\hline 13.6445 & 12.5669 & 6537 & 59.8703 \\
\hline 13.4717 & 12.5122 & 8816 & 58.6658 \\
\hline 13.4601 & 12.3814 & 1.0810 & 56.7397 \\
\hline 13.4271 & 12.3054 & 1.2946 & 55.8208 \\
\hline . & • & . & \\
\hline . & . & - & \\
\hline . & . & - & \\
\hline \#ЕOF & . & - & \\
\hline
\end{tabular}

A shoreline file may contain as many shoreline objects as needed. Objects may include islands, rivers, several lakes, etc. 


\section{GRAPHICAL USER INTERFACE (GUI)}

The Data_Animator user interface (GUI) was designed as drop down menus, each menu designed for data input, rendering of key frames, keyframe animations or full animations. The GUI also lets the user choose the topography and the bathymetry files to use, the fonts to use for titling, the number of colours (up to 256), the colours and the range of data each colour represents. Point-and-click mouse operations allow the user to manipulate many features, with immediate on-screen feedback. This GUI allows the user to quickly design an attractive animation (or single frame) to exact specifications.

\section{KeyFrame-Oriented Construction of Animations}

Animations are generated by defining 'keyframes' of known data, each located at a specific point in time. The program can then interpolate over time, between keyframes, to create smoothly animated transitions (in-between frames).

Animations are created in much the same way as movies are. Snapshots of action, called 'frames', are recorded at regular intervals in time. They are then displayed quickly one after the other to create the illusion of continuous movement. Computer animations are quite similar: they are a collection of still images of the computer screen which can be displayed very quickly, one after the other.

To create the illusion of smooth movement, the frames of an animation, or movie, must be displayed very quickly. For example, standard North American television screens display 30 still frames every second. This is also the standard rate for displaying animations on the Amiga computer. For example, an animation composed of 1800 still frames will play for one minute at the standard rate of 30 frames per second (fps).

This high rate of display presents a problem for visualizing scientific data. In most cases, the data provided is not nearly dense enough in time to generate such a large number of frames. Because of this, a method has been developed to 'stretch out' the data provided to the program.

Frames are categorized into two different types: KeyFrames, and in-between frames. 'KeyFrames' are those frames where known data values are provided by the user. These keyframes are created and manipulated by the user in the Data Animator program. A simple animation of only keyframes can be generated by the program, and if played back very slowly (eg. $1 \mathrm{fps)} \mathrm{it} \mathrm{will} \mathrm{provide} \mathrm{a} \mathrm{short,} \mathrm{jumpy}$ display of the data (like a slide show).

The 'in-between' frames are generated by the computer by interpolating over time between consecutive keyframes. Generation of in-between frames is the time consuming part of creating an animation. Each pixel on the screen has to be generated by linear interpolation in time (interpolation in space is used for key frames). In countries with the NTSC standard (e.g., North America and Japan), televisions display 30 frames per second, thus, to create an animation where each frame was rendered separately, an 1800 frames animation had to be developed (Of course it is also possible to play an animation at a slower rate, so that each NTSC frame corresponds to more than one rendered frame). To compute the number of in-between frames a simple proportion was made to accommodate these boundaries. In this way, the computer can insert frames of computed information between the keyframes of known data to create longer, smoother animations. The following options are available in Dat Animator:

\section{Option Description}


Single Frame

Key Frame
To render only the current keyframe.

To render multiple keyframes to an FLC file. When the Begin Rendering' command is issued, rendering will begin at the first keyframe and continue for the number of frames specified in the Number of Frames' input box.

Full
To render a full animation, including keyframes and interpolated inbetween frames, to an FLC file. The total number of frames rendered will match the value in the 'Number of Frames' input box. The number of in-between frames rendered between each pair of keyframes will be calculated based on the sample dates of the keyframes.

The GUI contains many commands to control the graphical settings.

1. Graph type

- plane, transect, 3D

2. Graph limits

- north, south east and west limits in degrees.minutes.seconds of latitude/longitude

- surface and bottom in meters

3. Graph view

- scale factors for each axis

4. Display options

- bounding box

- site markers, site numbers

- label depth axis

- shoreline

5. Interpolation

- Closest-Sites interpolation, as well as the number of closest sites to use in the interpolation calculations

6. Graph labels

- main title and colour legend title

- Date display options (any combination of day, month, year)

- Fonts for all labels. For video purposes, we recommend choosing a large and easily legible font. 
7. Colours

- number of colours to use (256 available)

- lowest data value of lowest colour

- data range for each colour

8. Animation type

- single frame, key frame or full animation

- number of frames to render

\section{Transect line}

- straight line defined by the user along which a transect is rendered. A transect line can be interactively chosen by moving the mouse on the screen to a location, start of the transect, and then to another to indicate the end of the transect. The computer program then identified the closest stations to each point in the transect line to create the desiderate visualization. The number of closest stations can be chosen by the user, usually this number ranges between two and five. This user-definable feature gives the program flexibility to deal with different data set densities. The closest stations, weighted by the inverse of the distance squared, are also used in the top view. Thus, all data visualizations are internally consistent.

Each menu command has an associated requester to change settings. If possible, any changes are reflected immediately in the main project window.

\section{ANIMATIONS}

Data Animator uses two interpolation methods and one assumption are used to create the animation. Vertical interpolation is done using a spline. Horizontally, the interpolation is linear, but non-linearly modified by the weights (inverse to the square of the distance from the station, as mentioned in the previous paragraph). Once the variable of interest is computed, a colour is assigned to each pixel to represent the variable at that location. A colour map on the right of the screen, describes the relation between the values of the variable and the colour. The user can decide the colour palette, i.e. which colour represents which range of data.

Figure 1 shows four frames out of an animation showing the behaviour of chlorophyll in Cootes Paradise from August 6 to August 25, 1986. To represent these frames in black and white rather than colour or grey scale, these frames have been edited for clarity (The original colour pictures and the full animation for 1986 are included in the ISESS1.ZIP file; The animations, in FLC format, can be obtained through anonymous FTP: ftp.cciw.ca, directory incoming/halfon). Animations for all water quality parameters (Total phosphorus, suspended solids, Secchi disk, etc.) for all subsequent years have also been performed. The interesting observation from this sequence is the presence of a gradient from west to east and a gradient from east to west, stations 1 to station 3 . Indeed station 3 has a concentration of chlorophyll lower than at stations at the west and the east. The animation show a dilution pattern originating in Hamilton Harbour and moving to the west. Conversely the damaging effects of the wastewater treatment plant in the west end stop in the west pond and do not influence the main part of 
Cootes Paradise. This sequence of events in 1986 is not immediately evident from the spreadsheet data, only a dynamic visualization of the data permitted this interpretation.

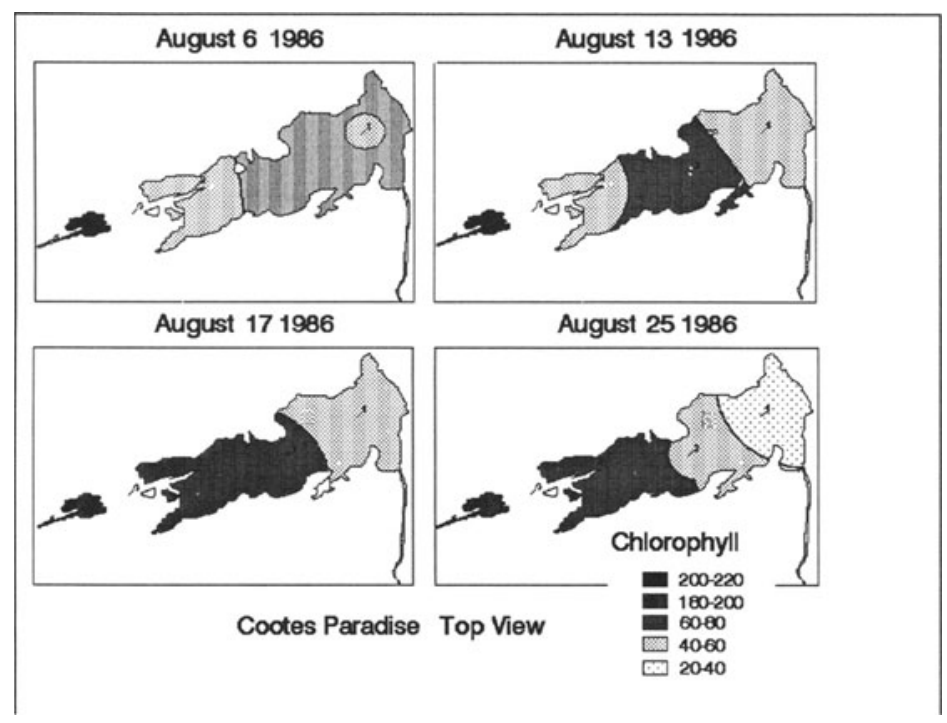

Figure 1 Four frames of the animation of chlorophyll concentration in Cootes Paradise, Lake Ontario are presented. The forth frame is a keyframe, i.e., data were used to create this frame. The other three frames are in-between frames created with interpolations in space and in time. The full colour animation is in FLC format and is posted (see text). 


\section{DISCUSSION}

In Cootes Paradise data have been collected for about a decade, at several stations a few times a year. The amount of data is large considering the fact that at each station many water quality variables were collected. Visualization permits a dynamic comparison of data collected over a season or over several years. Several animations can be run in different windows at the same time. Two dimensional displays are projections of the three dimensional data on one axis. A two dimensional display might be a transect or a top view. Visualization implies that the data collected at different stations and different times are interpolated in space and in time. Lake topography and lake bathymetry files are used to relate data collected in the lake(s) with topographical features.

Data_Animator was initially developed for use by the scientists who collected the data. Computer animation, computer graphics, video technology can also be used to present both to the general public and to specialized scientific audiences research result on aquatic environments. The use of animation can expand to include other scientific information and preparation of interactive information tools. The program runs on PCs and is optimized for Pentium chips.

\section{ACKNOWLEDGMENTS}

Dr. Pat Chow-Fraser kindly provided the 1986 chlorophyll data shown in Figure 1.

\section{REFERENCES}

Herman, G.T. and Liu, H.K. (1979) Three dimensional display of human organs from computed tomograms. Computer Graphics and Image Processing, 8, 1-21.

Halfon, E. ( Ed.) (1989) Microcomputers in ecological modelling: a special issue dedicated to research, education and computer graphics, Ecological Modelling, Vol. 47.

Halfon, E., Tartari, G., Brendon, D., and Binelli, A. (1993) Visualization and limnological interpretation of liming treatments in a deep lake, Lake Orta, Italy, using computer generated animations. In IFIP, WG 5.11 Working conference on Computer Support for Environmental Assessment, CSEIA '93, villa Olmo, Como, Oct. 6-8, 1993. pp. 82-91.

Halfon, E., Tartari, G., Howell, M., and Binelli, A. (1994) Visualization of pH changes due to liming treatments in Lake Orta, Italy, using computer generated animations. In G. Guariso and B. Page (eds) Computer Support for Environmental Assessment, Elsevier Science, 37-48.

Halfon, E. and Howell, M. (1995) Visualization of limnological data as two- and three-dimensional computer generated animations. J. Biol. Systems , 4, 3-25.

Halfon, E. and Tartari, G. (1995) Visualizzazione di dati raccolti nel Lago d'Orta negli anni 1989-1991. Acqua e Aria (in press).

Halfon, E. (ed) (1995) Special issue on the use of computer graphics and visualization. J. Biological System s, Vol. 2. 
Kaufmann, A. (1991) Volume Visualization, IEEE Computer Society Press, Los Alamitos, Ca., 487 pp.

Sunguroff, A. and Greenberg, D. (1978) Computer generated images for medical applications. Computer Graphics, 12, 196-202.

\section{BIOGRAPHY}

I am a research scientist with Environment Canada. I am also a professor in the Biology Department at McMaster University in Hamilton, Ontario. My interests are in scientific visualization, mathematical modelling of the fate of toxic contaminants, risk assessment of toxic contaminants and their ranking. 